Pacific

Journal of

Mathematics

THE CONCAVITY OF THE GAUSSIAN CURVATURE OF THE CONVEX LEVEL SETS OF MINIMAL SURFACES WITH RESPECT TO THE HEIGHT

PEI-He WANG

Volume $267 \quad$ No. 2

February 2014 


\title{
THE CONCAVITY OF THE GAUSSIAN CURVATURE OF THE CONVEX LEVEL SETS OF MINIMAL SURFACES WITH RESPECT TO THE HEIGHT
}

\author{
PEI-HE WANG
}

\begin{abstract}
For the minimal graph with strictly convex level sets, we find an auxiliary function to study the Gaussian curvature of the level sets. We prove that this curvature function is a concave function with respect to the height of the minimal surface while this auxiliary function is almost sharp when the minimal surface is the catenoid.
\end{abstract}

\section{Introduction}

Consider a function whose graph is minimal and whose level sets are strictly convex. Extending work of Longinetti [1987], we explore the relation between the Gaussian curvature of the level sets and the height.

The nature of the level sets of the solutions of elliptic partial differential equations is a subject with a long history, going back to results of Shiffman in the 1950s for minimal surfaces. The curvature of such level sets has also been studied for several decades. Some key contributions to these problems are listed in the introduction of [Chen and Shi 2011]. Here we just mention some recent developments directly relevant to our problem.

Jost, Ma, and Ou [Jost et al. 2012] and Ma, Ye, and Ye [Ma et al. 2011] proved that the Gaussian and principal curvatures of convex level sets of three-dimensional harmonic functions attain their minima on the boundary. Ma, Ou, and Zhang [2010] gave estimates of the Gaussian curvature of convex level sets of higher-dimensional harmonic functions based on the Gaussian curvature of the boundary and the norm of the gradient on the boundary. Wang and Zhang [2012] have given estimates for the Gaussian curvature of convex level sets of minimal surfaces, Poisson equations, and a class of semilinear elliptic partial differential equations studied by Caffarelli and Spruck [1982].

Research was supported by STPF of University (number J11LA05), NSFC (number ZR2012AM010), the Postdoctoral fund (number 201203030) of Shandong Province and the Postdoctoral Fund (number 2012M521302) of China.

MSC2010: 35B45.

Keywords: concavity, minimal surface, Gaussian curvature, level sets. 
In this paper we use the support function of strictly convex level sets and the maximum principle to obtain the concavity of the Gaussian curvature of convex level sets of minimal graphs with respect to the height:

Theorem 1.1. Let $\Omega$ be a bounded smooth domain in $\mathbb{R}^{n}, n \geq 2$, and let

$$
u \in C^{4}(\Omega) \cap C^{2}(\bar{\Omega}), \quad t_{0} \leq u(x) \leq t_{1}
$$

be a minimal graph in $\Omega$, that is, one such that

$$
\operatorname{div} \frac{\nabla u}{\sqrt{1+|\nabla u|^{2}}}=0 \quad \text { in } \Omega .
$$

Assume $|\nabla u| \neq 0$ in $\bar{\Omega}$. Let

$$
\Gamma_{t}=\{x \in \Omega: u(x)=t\} \quad \text { for } t_{0}<t<t_{1}
$$

be the level sets of $u$ and let $K$ be their Gaussian curvature function. For

$$
f(t)=\min \left\{\left[\left(\frac{|\nabla u|^{2}}{1+|\nabla u|^{2}}\right)^{\frac{n-3}{2}} K\right]^{\frac{1}{n-1}}(x): x \in \Gamma_{t}\right\},
$$

if the level sets of $u$ are strictly convex with respect to the normal $\nabla u$, we have the differential inequality

$$
D^{2} f(t) \leq 0 \quad \text { in }\left(t_{0}, t_{1}\right)
$$

Under the same assumption as in Theorem 1.1, Wang and Zhang [2012] proved the following statement: for $n \geq 2$, the function $\left(|\nabla u|^{2} /\left(1+|\nabla u|^{2}\right)\right)^{\theta} K$ attains its minimum on the boundary, where $\theta=-\frac{1}{2}$ or $\theta \geq \frac{1}{2}(n-3)$. From this fact they got the lower bound estimates for the Gaussian curvature of the level sets.

Corollary 1.2. Let u satisfy

$$
\begin{cases}\operatorname{div} \frac{\nabla u}{\sqrt{1+|\nabla u|^{2}}}=0 & \text { in } \Omega=\Omega_{0} \backslash \bar{\Omega}_{1}, \\ u=0 & \text { on } \partial \Omega_{0}, \\ u=1 & \text { on } \partial \Omega_{1},\end{cases}
$$

where $\Omega_{0}$ and $\Omega_{1}$ are bounded smooth convex domains in $\mathbb{R}^{n}, n \geq 2, \bar{\Omega}_{1} \subset \Omega_{0}$. Assume $|\nabla u| \neq 0$ in $\bar{\Omega}$ and the level sets of $u$ are strictly convex with respect to normal $\nabla u$. Let $K$ be the Gaussian curvature of the level sets. For any point $x \in \Gamma_{t}, 0<t<1$, we have the following estimates.

- For $n=3$, we have

$$
K(x)^{1 / 2} \geq(1-t)\left(\min _{\partial \Omega_{0}} K\right)^{1 / 2}+t\left(\min _{\partial \Omega_{1}} K\right)^{1 / 2} .
$$


- For $n \neq 3$, we have

$$
\begin{aligned}
& {\left[\left(\frac{|\nabla u|^{2}}{1+|\nabla u|^{2}}\right)^{\frac{n-3}{2}} K\right]^{\frac{1}{n-1}}(x)} \\
& \geq(1-t) \min _{\partial \Omega_{0}}\left[\left(\frac{|\nabla u|^{2}}{1+|\nabla u|^{2}}\right)^{\frac{n-3}{2}} K\right]^{\frac{1}{n-1}}+t \min _{\partial \Omega_{1}}\left[\left(\frac{|\nabla u|^{2}}{1+|\nabla u|^{2}}\right)^{\frac{n-3}{2}} K\right]^{\frac{1}{n-1}} .
\end{aligned}
$$

Remark 1.3. The following example shows that our estimates are almost sharp in a sense. Let $u(r, \theta), r>2$, be the $n$-dimensional catenoid:

$$
u(r, \theta)=\int_{-r}^{-2} \frac{1}{\sqrt{s^{2(n-1)}-1}} d s .
$$

Then

$$
|\nabla u|=\frac{1}{\sqrt{r^{2(n-1)}-1}},
$$

and the Gaussian curvature of the level set at $x$ is $K(x)=r^{1-n}$. Hence,

$$
f(t)=\left[\left(\frac{|\nabla u|^{2}}{1+|\nabla u|^{2}}\right)^{\frac{n-3}{2}} K\right]^{\frac{1}{n-1}}=r^{2-n} .
$$

For $n=2, f(t)$ becomes a constant function, which shows that our estimate of its concavity is sharp. Now we turn to the case $n>2$.

Set

$$
R=\int_{-\infty}^{-2} \frac{1}{\sqrt{s^{2(n-1)}-1}} d s
$$

Then we have

$$
\begin{aligned}
-u+R & =\int_{-\infty}^{-r} \frac{1}{s^{n-1}} d s-\int_{-\infty}^{-r} \frac{1}{s^{n-1}}\left[1-\frac{1}{\sqrt{1-s^{-2(n-1)}}}\right] d s \\
& =\frac{(-1)^{n}}{2-n} r^{2-n}+\mathcal{O}\left(r^{4-3 n}\right) .
\end{aligned}
$$

This means that

$$
f(t)=(-1)^{n}(2-n)(R-t)+O\left(r^{4-3 n}\right),
$$

which shows the "almost sharpness" of our estimate in higher dimensions.

To prove these theorems, let $K$ be the Gaussian curvature of the convex level sets, and let $\varphi=\log K(x)+\rho\left(|\nabla u|^{2}\right)$. For suitable choices of $\rho$ and $\beta$, we shall show the elliptic differential inequality

$$
L\left(e^{\beta \varphi}\right) \leq 0 \quad \bmod \nabla_{\theta} \varphi \quad \text { in } \Omega,
$$


where $L$ is the elliptic operator associated with the equation we discussed and here we have suppressed the terms involving $\nabla_{\theta} \varphi$ (see the notations below) with locally bounded coefficients. Then we apply the strong minimum principle to obtain the main results.

In Section 2, we first give brief definitions on the support function of the level sets, and then we obtain the equation of the minimal graph in terms of the support function. We prove Theorem 1.1 in Section 3 by formal calculations. The main technique in the proof consists of rearranging the second and third derivative terms using the equation and the first derivative condition for $\varphi$. The key idea is Pogorelov's method in a priori estimates for fully nonlinear elliptic equations.

\section{Notations and preliminaries}

Let $\Omega_{0}$ and $\Omega_{1}$ be bounded smooth open convex subsets of $\mathbb{R}^{n}$ such that $\bar{\Omega}_{1} \subset \Omega_{0}$, and let $\Omega=\Omega_{0} \backslash \bar{\Omega}_{1}$. Let $u: \bar{\Omega} \rightarrow \mathbb{R}$ be a smooth function with $|D u|>0$ in $\Omega$ and let its level sets be strictly convex with respect to the normal direction $D u$.

For simplicity, we will assume that

$$
\begin{array}{ll}
u=0 & \text { on } \partial \Omega_{0}, \\
u=1 & \text { on } \partial \Omega_{1},
\end{array}
$$

and we extend $u$ to $\Omega_{1}$ with the value 1 . For $0 \leq t \leq 1$, we set

$$
\bar{\Omega}_{t}=\left\{x \in \bar{\Omega}_{0}: u \geq t\right\} ;
$$

Then every $x \in \Omega$ belongs to the boundary of $\bar{\Omega}_{u(x)}$.

Next we define the support function of $u$, denoted by

$$
H: \mathbb{R}^{n} \times[0,1] \rightarrow \mathbb{R}
$$

as follows: for each $t \in[0,1], H(\cdot, t)$ is the support function of the convex body $\bar{\Omega}_{t}$, that is,

$$
H(X, t)=H_{\bar{\Omega}_{t}}(X) \quad \text { for all } X \in \mathbb{R}^{n}, t \in[0,1] .
$$

For details, see [Colesanti and Salani 2003; Longinetti and Salani 2007].

The rest of this section is devoted to deriving the minimal graph by means of the support function. For this we need a reformulation of the first and second derivatives of $u$ in terms of the support function $h_{\Omega_{t}}$, which is the restriction of $H(\cdot, t)$ to the unit sphere $\mathbb{S}^{n-1}$; see [Chiti and Longinetti 1992; Longinetti and Salani 2007]. For the convenience of the reader, we report the main steps here.

Recall that $h$ is the restriction of $H$ to $\mathbb{S}^{n-1} \times[0,1]$, so $h(\theta, t)=H(Y(\theta), t)=$ $h_{\bar{\Omega}_{t}}(Y(\theta))$ where $t \in[0,1]$ and $Y(\theta) \in \mathbb{S}^{n}$ is a unit vector with coordinate $\theta$. Since the level sets of $u$ are strictly convex and $h(\theta, t)$ is well defined, the map

$$
x(X, t)=x_{\bar{\Omega}_{t}}(X),
$$


which assigns to every $(X, t) \in \mathbb{R}^{n} \backslash\{0\} \times(0,1)$ the unique point $x \in \Omega$ on the level surface $\{u=t\}$, where the gradient of $u$ is parallel to $X$ (and orientation reversed).

Let

$$
T_{i}=\frac{\partial Y}{\partial \theta_{i}},
$$

so that $\left\{T_{1}, \ldots, T_{n-1}\right\}$ is a tangent frame field on $\mathbb{S}^{n-1}$, and let

$$
x(\theta, t)=x_{\bar{\Omega}_{t}}(Y(\theta)) ;
$$

we denote its inverse map by

$$
v:\left(x_{1}, \ldots, x_{n}\right) \rightarrow\left(\theta_{1}, \ldots, \theta_{n-1}, t\right) .
$$

Notice that all these maps ( $h, x$, and $v$ ) depend on the considered function $u$ (like $H)$, even if we do not adopt any explicit notation to stress this fact.

For $h(\theta, t)=\langle x(\theta, t), Y(\theta)\rangle$, since $Y$ is orthogonal to $\partial \bar{\Omega}_{t}$ at $x(\theta, t)$, deriving the previous equation, we obtain

$$
h_{i}=\left\langle x, T_{i}\right\rangle .
$$

In order to simplify some computations, we can also assume that $\theta_{1}, \ldots, \theta_{n-1}, Y$ is an orthonormal frame positively oriented. Hence, from the previous two equalities, we have

and

$$
x=h Y+\sum_{i} h_{i} T_{i}
$$

$$
\frac{\partial T_{i}}{\partial \theta_{j}}=-\delta_{i j} Y \quad \text { at } x
$$

where the summation index runs from 1 to $n-1$ if no extra explanation is given, and $\delta_{i j}$ is the standard Kronecker symbol. Following [Chiti and Longinetti 1992], we obtain, at the point $x$ under consideration,

$$
\begin{aligned}
& \frac{\partial x}{\partial t}=h_{t} Y+\sum_{i} h_{t i} T_{i}, \\
& \frac{\partial x}{\partial \theta_{j}}=h T_{j}+\sum_{i} h_{i j} T_{i}, \quad j=1, \ldots, n-1 .
\end{aligned}
$$

The inverse of the above Jacobian matrix is

$$
\begin{array}{lll}
\frac{\partial t}{\partial x_{\alpha}} & =h_{t}^{-1}[Y]_{\alpha}, & \alpha=1, \ldots, n, \\
\frac{\partial \theta_{i}}{\partial x_{\alpha}}=\sum_{j} b^{i j}\left[T_{j}-h_{t}^{-1} h_{t j} Y\right]_{\alpha}, & \alpha=1, \ldots, n,
\end{array}
$$


where $[\cdot]_{i}$ denotes the $i$-coordinate of the vector in the bracket and

$$
b_{i j}=\left\langle\frac{\partial x}{\partial \theta_{i}}, \frac{\partial Y}{\partial \theta_{j}}\right\rangle=h \delta_{i j}+h_{i j}
$$

denotes the inverse tensor of the second fundamental form of the level surface $\partial \bar{\Omega}_{t}$ at $x(\theta, t)$. The eigenvalues of the tensor $b^{i j}$ are the principal curvatures $\kappa_{1}, \ldots, \kappa_{n-1}$ of $\partial \bar{\Omega}_{t}$ at $x(\theta, t)$; see [Schneider 1993].

The first equation of (2-1) can be rewritten as

$$
D u=\frac{Y}{h_{t}},
$$

where the left hand side is computed at $x(\theta, t)$, while the right hand side is computed at $(\theta, t)$. It follows that

$$
|D u|=-\frac{1}{h_{t}} .
$$

By the chain rule and (2-1), the second derivatives of $u$ in terms of $h$ can be computed as

$$
u_{\alpha \beta}=\sum_{i, j}\left[-h_{t}^{-2} h_{t i} Y+h_{t}^{-1} T_{i}\right]_{\alpha} b^{i j}\left[T_{j}-h_{t}^{-1} h_{t j} Y\right]_{\beta}-h_{t}^{-3} h_{t t}[Y]_{\alpha}[Y]_{\beta}
$$

for $\alpha, \beta=1, \ldots, n$.

In these new coordinates, the minimal graph equation, $\operatorname{div} \frac{\nabla u}{\sqrt{1+|\nabla u|^{2}}}=0$, reads

$$
h_{t t}=\sum_{i, j}\left[\left(1+h_{t}^{2}\right) \delta_{i j}+h_{t i} h_{t j}\right] b^{i j},
$$

and the associated linear elliptic operator is

$$
L=\sum_{i, j, p, q}\left[\left(1+h_{t}^{2}\right) \delta_{p q}+h_{t p} h_{t q}\right] b^{i p} b^{j q} \frac{\partial^{2}}{\partial \theta_{i} \partial \theta_{j}}-2 \sum_{i, j} h_{t j} b^{i j} \frac{\partial^{2}}{\partial \theta_{i} \partial t}+\frac{\partial^{2}}{\partial t^{2}}
$$

Now we recall the well-known commutation formulas for the covariant derivatives of a smooth function $u \in C^{4}\left(S^{n}\right)$.

$$
\begin{aligned}
u_{i j k}-u_{i k j} & =-u_{k} \delta_{i j}+u_{j} \delta_{i k}, \\
u_{i j k l}-u_{i j l k} & =u_{i k} \delta_{j l}-u_{i l} \delta_{j k}+u_{k j} \delta_{i l}-u_{l j} \delta_{i k} .
\end{aligned}
$$

They will be used during the calculations in the next section. By the definition of $b_{i j}$ and the above commutation formulas, we easily get the following Codazzi-type formula:

$$
b_{i j, k}=b_{i k, j} .
$$




\section{Gauss curvature of the level sets of minimal graph}

In this section we prove Theorem 1.1. We state a technical lemma.

Lemma 3.1 [Ma et al. 2010]. Let $\lambda \geq 0, \mu \in \mathbb{R}, b_{k}>0$, and $c_{k} \in \mathbb{R}$ for $2 \leq k \leq n-1$. Define the quadratic polynomial

$$
Q\left(X_{2}, \ldots, X_{n-1}\right)=-\sum_{2 \leq k \leq n-1} b_{k} X_{k}^{2}-\lambda\left(\sum_{2 \leq k \leq n-1} X_{k}\right)^{2}+4 \mu \sum_{2 \leq k \leq n-1} c_{k} X_{k} .
$$

Then we have

$$
Q\left(X_{2}, \ldots, X_{n-1}\right) \leq 4 \mu^{2} \Gamma,
$$

where

$$
\Gamma=\sum_{2 \leq k \leq n-1} \frac{c_{k}^{2}}{b_{k}}-\lambda\left(1+\lambda \sum_{2 \leq k \leq n-1} \frac{1}{b_{k}}\right)^{-1}\left(\sum_{2 \leq k \leq n-1} \frac{c_{k}}{b_{k}}\right)^{2} .
$$

For a continuous function $f(t)$ on $[0,1]$, we define its generalized second-order derivative at any point $t$ in $(0,1)$ as

$$
D^{2} f(t)=\limsup _{h \rightarrow 0} \frac{f(t+h)+f(t-h)-2 f(t)}{h^{2}} .
$$

Let $B$ be the quotient set $B \equiv \mathbb{R}^{n} / 2 \pi \mathbb{Z}^{n}$ and let $Q \equiv B \times(0,1)$. Let $G(\theta, t)$ be a regular function in $Q$ such that $\mathscr{L}(G(\theta, t)) \geq 0$ for $(\theta, t) \in Q$, where $\mathscr{L}$ is an elliptic operator of the form

$$
\mathscr{L}=\sum_{i, j} a^{i j} \frac{\partial^{2}}{\partial \theta_{i} \partial \theta_{j}}+\sum_{i} b^{i} \frac{\partial^{2}}{\partial \theta_{i} \partial t}+\frac{\partial^{2}}{\partial t^{2}}+\sum_{i} c^{i} \frac{\partial}{\partial \theta_{i}}
$$

with regular coefficients $a^{i j}, b^{i}, c^{i}$.

Lemma 3.2 [Longinetti 1987]. The function $\phi(t)=\max \{G(\theta, t): \theta \in B\}$ satisfies the differential inequality

$$
D^{2} \phi(t) \geq 0 \text {. }
$$

Moreover, $\phi(t)$ is a convex function with respect to $t$.

The lemma is proved only in dimension $n=2$ in [Longinetti 1987], but it is easy to see that it is valid for the general case $n \geq 2$.

Since the level sets of $u$ are strictly convex with respect to the normal $D u$, the matrix of second fundamental form $\left(b_{i j}\right)$ is positive definite in $\Omega$. Set

$$
\varphi=\rho\left(h_{t}^{2}\right)-\log K(x),
$$

where $K=\operatorname{det}\left(b^{i j}\right)$ is the Gaussian curvature of the level sets and $\rho(t)$ is a smooth function defined on $(0,+\infty)$. For suitable choices of $\rho$ and $\beta$, we will derive the 
differential inequality

$$
L\left(e^{\beta \varphi}\right) \leq 0 \quad \bmod \nabla_{\theta} \varphi \quad \text { in } \Omega,
$$

where the elliptic operator $L$ is given in (2-5) and we have modified the terms involving $\nabla_{\theta} \varphi$ with locally bounded coefficients. Then, by applying a maximum principle argument in Lemma 3.2, we can obtain the desired result.

In order to prove (3-1) at an arbitrary point $x_{0} \in \Omega$, we may assume the matrix $\left(b_{i j}\left(x_{0}\right)\right)$ is diagonal by rotating the coordinate system suitably. From now on, all the calculations will be done at the fixed point $x_{0}$.

Proof of Theorem 1.1. We shall prove the theorem in three steps.

Step 1: computation $L(\varphi)$. Taking the first derivative of $\varphi$, we get

$$
\begin{aligned}
\frac{\partial \varphi}{\partial \theta_{j}} & =2 \rho^{\prime} h_{t} h_{t j}+\sum_{k, l} b^{k l} b_{k l, j}, \\
\frac{\partial \varphi}{\partial t} & =2 \rho^{\prime} h_{t} h_{t t}+\sum_{k, l} b^{k l} b_{k l, t} .
\end{aligned}
$$

Taking the derivative of (3-2) and (3-3) once more, we have

$$
\begin{aligned}
\frac{\partial^{2} \varphi}{\partial \theta_{i} \partial \theta_{j}} & =\left(2 \rho^{\prime}+4 \rho^{\prime \prime} h_{t}^{2}\right) h_{t i} h_{t j}+2 \rho^{\prime} h_{t} h_{t j i}-\sum_{k, l, r, s} b^{k r} b_{r s, i} b^{s l} b_{k l, j}+\sum_{k, l} b^{k l} b_{k l, j i}, \\
\frac{\partial^{2} \varphi}{\partial \theta_{i} \partial t} & =\left(2 \rho^{\prime}+4 \rho^{\prime \prime} h_{t}^{2}\right) h_{t i} h_{t t}+2 \rho^{\prime} h_{t} h_{t t i}-\sum_{k, l, r, s} b^{k r} b_{r s, i} b^{s l} b_{k l, t}+\sum_{k, l} b^{k l} b_{k l, t i}, \\
\frac{\partial^{2} \varphi}{\partial t^{2}} & =\left(2 \rho^{\prime}+4 \rho^{\prime \prime} h_{t}^{2}\right) h_{t t}^{2}+2 \rho^{\prime} h_{t} h_{t t t}-\sum_{k, l, r, s} b^{k r} b_{r s, t} b^{s l} b_{k l, t}+\sum_{k, l} b^{k l} b_{k l, t t} .
\end{aligned}
$$

So we can wrtie

$$
L(\varphi)=I_{1}+I_{2}+I_{3}+I_{4},
$$

with

$$
\begin{aligned}
& I_{1}=\left(2 \rho^{\prime}+4 \rho^{\prime \prime} h_{t}^{2}\right)\left[\sum_{i, j}\left[\left(1+h_{t}^{2}\right) \delta_{i j}+h_{t i} h_{t j}\right] b^{i i} b^{j j} h_{t i} h_{t j}-2 \sum_{i} h_{t i}^{2} b^{i i} h_{t t}+h_{t t}^{2}\right], \\
& I_{2}=2 \rho^{\prime} h_{t}\left[\sum_{i, j}\left[\left(1+h_{t}^{2}\right) \delta_{i j}+h_{t i} h_{t j}\right] b^{i i} b^{j j} h_{t j i}-2 \sum_{i} h_{t i} b^{i i} h_{t t i}+h_{t t t}\right], \\
& I_{3}=-\sum_{k, l} b^{k k} b^{l l}\left[\sum_{i, j}\left[\left(1+h_{t}^{2}\right) \delta_{i j}+h_{t i} h_{t j}\right] b^{i i} b^{j j} b_{k l, i} b_{k l, j},-2 \sum_{i} h_{t i} b^{i i} b_{k l, i} b_{k l, t}\right. \\
& I_{4}=\sum_{k} b^{k k} L\left(b_{k k}\right) .
\end{aligned}
$$


In the rest of this section, we will deal with the four terms above respectively. For the term $I_{1}$, by recalling our equation, that is,

$$
h_{t t}=\sum_{i, j}\left[\left(1+h_{t}^{2}\right) \delta_{i j}+h_{t i} h_{t j}\right] b^{i j},
$$

we have, by recalling that $\left(b^{i j}\right)$ is diagonal at $x_{0}$,

$$
\begin{aligned}
I_{1} & =\left(2 \rho^{\prime}+4 \rho^{\prime \prime} h_{t}^{2}\right)\left[\sum_{i, j}\left[\left(1+h_{t}^{2}\right) \delta_{i j}+h_{t i} h_{t j}\right] b^{i i} b^{j j} h_{t i} h_{t j}-2 \sum_{i} h_{t i}^{2} b^{i i} h_{t t}+h_{t t}^{2}\right] \\
& =\left(2 \rho^{\prime}+4 \rho^{\prime \prime} h_{t}^{2}\right)\left[\left(1+h_{t}^{2}\right) \sum_{i}\left(h_{t i} b^{i i}\right)^{2}+\left(\sum_{i} h_{t i}^{2} b^{i i}-h_{t t}\right)^{2}\right] \\
& =\left(2 \rho^{\prime}+4 \rho^{\prime \prime} h_{t}^{2}\right)\left(1+h_{t}^{2}\right) \sum_{i}\left(h_{t i} b^{i i}\right)^{2}+\left(2 \rho^{\prime}+4 \rho^{\prime \prime} h_{t}^{2}\right)\left(1+h_{t}^{2}\right)^{2} \sigma_{1}^{2},
\end{aligned}
$$

where $\sigma_{1}=\sum_{i} b^{i i}$ is the mean curvature.

Now we treat the term $I_{2}$. Differentiating (3-5) with respect to $t$, we have

$$
h_{t t t}=2 h_{t} h_{t t} \sigma_{1}+2 \sum_{i, j} h_{t t i} h_{t j} b^{i j}-\sum_{i, j}\left[\left(1+h_{t}^{2}\right) \delta_{i j}+h_{t i} h_{t j}\right] b^{i i} b^{j j} b_{i j, t} .
$$

By inserting (3-7) into $I_{2}$, we can get

$$
\begin{aligned}
I_{2} & =2 \rho^{\prime} h_{t}\left[\sum_{i, j}\left[\left(1+h_{t}^{2}\right) \delta_{i j}+h_{t i} h_{t j}\right] b^{i i} b^{j j} h_{t j i}-2 \sum_{i} h_{t i} b^{i i} h_{t t i}+h_{t t t}\right] \\
& =2 \rho^{\prime} h_{t}\left[\sum_{i, j}\left[\left(1+h_{t}^{2}\right) \delta_{i j}+h_{t i} h_{t j}\right] b^{i i} b^{j j}\left(h_{t j i}-b_{i j, t}\right)+2 h_{t} h_{t t} \sigma_{1}\right] .
\end{aligned}
$$

Recalling the definition of the second fundamental form, that is, (2-2), together with (3-5), we obtain

$$
\begin{aligned}
I_{2} & =2 \rho^{\prime} h_{t}\left[\sum_{i, j}\left[\left(1+h_{t}^{2}\right) \delta_{i j}+h_{t i} h_{t j}\right] b^{i i} b^{j j}\left(-h_{t} \delta_{i j}\right)+2 h_{t} h_{t t} \sigma_{1}\right] \\
& =-2 \rho^{\prime} h_{t}^{2}\left(1+h_{t}^{2}\right) \sum_{i}\left(b^{i i}\right)^{2}-2 \rho^{\prime} h_{t}^{2} \sum_{i}\left(h_{t i} b^{i i}\right)^{2}+4 \rho^{\prime} h_{t}^{2}\left(1+h_{t}^{2}\right) \sigma_{1}^{2} \\
& +4 \rho^{\prime} h_{t}^{2} \sigma_{1} \sum_{i} h_{t i}^{2} b^{i i} .
\end{aligned}
$$

Combining (3-6) and (3-8),

$$
\begin{aligned}
I_{1}+ & I_{2} \\
= & 4 \rho^{\prime} h_{t}^{2} \sigma_{1} \sum_{i} h_{t i}^{2} b^{i i}+\left[4 \rho^{\prime} h_{t}^{2}\left(1+h_{t}^{2}\right)+\left(2 \rho^{\prime}+4 \rho^{\prime \prime} h_{t}^{2}\right)\left(1+h_{t}^{2}\right)^{2}\right] \sigma_{1}^{2} \\
& +\left[\left(2 \rho^{\prime}+4 \rho^{\prime \prime} h_{t}^{2}\right)\left(1+h_{t}^{2}\right)-2 \rho^{\prime} h_{t}^{2}\right] \sum_{i}\left(h_{t i} b^{i i}\right)^{2}-2 \rho^{\prime} h_{t}^{2}\left(1+h_{t}^{2}\right) \sum_{i}\left(b^{i i}\right)^{2} .
\end{aligned}
$$


In order to deal with the last two terms, we shall compute $L\left(b_{k k}\right)$ in advance. In this process, the index $k$ is not summed. By differentiating (3-5) twice with respect to $\theta_{k}$, we have

$$
h_{t t k k}=J_{1}+J_{2}+J_{3}+J_{4},
$$

with

$$
\begin{aligned}
& J_{1}=\sum_{i, j}\left[\left(1+h_{t}^{2}\right) \delta_{i j}+h_{t i} h_{t j}\right]_{k k} b^{i j}, \\
& J_{2}=2 \sum_{i j, p, q}\left[\left(1+h_{t}^{2}\right) \delta_{i j}+h_{t i} h_{t j}\right]_{k}\left(-b^{i p} b_{p q, k} b^{q j}\right), \\
& J_{3}=\sum_{i j, p, q, r, s}\left[\left(1+h_{t}^{2}\right) \delta_{i j}+h_{t i} h_{t j}\right]\left(2 b^{i r} b_{r s, k} b^{s p} b_{p q, k} b^{q j}\right), \\
& J_{4}=\sum_{i j, p, q}\left[\left(1+h_{t}^{2}\right) \delta_{i j}+h_{t i} h_{t j}\right]\left(-b^{i p} b_{p q, k k} b^{q j}\right) .
\end{aligned}
$$

For the term $J_{1}$, we have

$$
\begin{aligned}
J_{1} & =\sum_{i, j}\left(2 h_{t} h_{t k} \delta_{i j}+h_{t i k} h_{t j}+h_{t i} h_{t j k}\right)_{k} b^{i j} \\
& =2 h_{t k}^{2} \sigma_{1}+2 h_{t} h_{t k k} \sigma_{1}+2 \sum_{i} h_{t i k k} h_{t i} b^{i i}+2 \sum_{i} h_{t i k}^{2} b^{i i} .
\end{aligned}
$$

Noticing that

$$
\begin{aligned}
h_{t i k} & =h_{k i t}=b_{k i, t}-h_{t} \delta_{k i}, \\
h_{t i k k} & =h_{i k k t}=b_{i k, k t}-h_{k t} \delta_{i k}=b_{k k, i t}-h_{k t} \delta_{i k},
\end{aligned}
$$

we obtain

$$
\begin{aligned}
J_{1}=2 h_{t k}^{2} \sigma_{1}+2 h_{t} b_{k k, t} \sigma_{1} & -2 h_{t}^{2} \sigma_{1}+2 \sum_{i} b_{k k, i t} h_{t i} b^{i i} \\
& -2 h_{t k}^{2} b^{k k}+2 \sum_{l} b_{k l, t}^{2} b^{l l}-4 h_{t} b_{k k, t} b^{k k}+2 h_{t}^{2} b^{k k} .
\end{aligned}
$$

For the term $J_{2}$, we have

$$
\begin{aligned}
J_{2} & =2 \sum_{i, j}\left(2 h_{t} h_{t k} \delta_{i j}+h_{t i k} h_{t j}+h_{t i} h_{t j k}\right)\left(-b^{i i} b_{i j, k} b^{j j}\right) \\
& =-4 h_{t} h_{t k} \sum_{i}\left(b^{i i}\right)^{2} b_{i i, k}-4 \sum_{i, j} h_{t i k} h_{t j} b^{i i} b^{j j} b_{i j, k} \\
= & -4 h_{t} h_{t k} \sum_{i}\left(b^{i i}\right)^{2} b_{i i, k}-4 \sum_{i, l} h_{t i} b^{i i} b^{l l} b_{k l, i} b_{k l, t} \\
& +4 h_{t} \sum_{j} h_{t j} b^{k k} b^{j j} b_{k k, j} .
\end{aligned}
$$


Note that we have changed the lower index during the above calculations and this will happen frequently in the following procedure.

Also we have

$$
J_{3}=2 \sum_{i, j, l}\left[\left(1+h_{t}^{2}\right) \delta_{i j}+h_{t i} h_{t j}\right] b^{i i} b^{j j} b^{l l} b_{k l, i} b_{k l, j}
$$

Applying the commutation rule $b_{i j, k l}-b_{i j, l k}=b_{j k} \delta_{i l}-b_{j l} \delta_{i k}+b_{i k} \delta_{j l}-b_{i l} \delta_{j k}$, for the term $J_{4}$, we have

$$
\begin{aligned}
J_{4} & =-\sum_{i, j}\left[\left(1+h_{t}^{2}\right) \delta_{i j}+h_{t i} h_{t j}\right] b^{i i} b^{j j} b_{i j, k k} \\
& =-\sum_{i, j}\left[\left(1+h_{t}^{2}\right) \delta_{i j}+h_{t i} h_{t j}\right] b^{i i} b^{j j}\left(b_{k k, i j}+b_{i j}-b_{k k} \delta_{i j}\right) .
\end{aligned}
$$

On the other hand,

$$
h_{t t k k}=h_{k k t t}=b_{k k, t t}-h_{t t}=b_{k k, t t}-\sum_{i, j}\left[\left(1+h_{t}^{2}\right) \delta_{i j}+h_{t i} h_{t j}\right] b^{i j} .
$$

By putting (3-11)-(3-15) into (3-10), recalling the definition of the operator $L$, we obtain

$$
\begin{aligned}
L\left(b_{k k}\right)= & \sum_{i, j}\left[\left(1+h_{t}^{2}\right) \delta_{i j}+h_{t i} h_{t j}\right] b^{i j}+2 h_{t k}^{2} \sigma_{1}+2 h_{t} b_{k k, t} \sigma_{1}-2 h_{t}^{2} \sigma_{1} \\
& -2 h_{t k}^{2} b^{k k}+2 \sum_{l} b_{k l, t}^{2} b^{l l}-4 h_{t} b^{k k} b_{k k, t}+2 h_{t}^{2} b^{k k}-4 h_{t} h_{t k} \sum_{i}\left(b^{i i}\right)^{2} b_{i i, k} \\
& -4 \sum_{i, l} h_{t i} b^{i i} b^{l l} b_{k l, i} b_{k l, t}+2 \sum_{i, j, l}\left[\left(1+h_{t}^{2}\right) \delta_{i j}+h_{t i} h_{t j}\right] b^{i i} b^{j j} b^{l l} b_{k l, i} b_{k l, j} \\
& +4 h_{t} \sum_{i} h_{t i} b^{k k} b^{i i} b_{k k, i}-\sum_{i, j}\left[\left(1+h_{t}^{2}\right) \delta_{i j}+h_{t i} h_{t j}\right] b^{i i} b^{j j}\left(b_{i j}-b_{k k} \delta_{i j}\right) .
\end{aligned}
$$

Therefore,

$$
\begin{array}{r}
I_{4}=2 \sum_{i, j, k, l}\left[\left(1+h_{t}^{2}\right) \delta_{i j}+h_{t i} h_{t j}\right] b^{i i} b^{j j} b^{k k} b^{l l} b_{k l, i} b_{k l, j}-4 \sum_{i, k, l} h_{t i} b^{i i} b^{k k} b^{l l} b_{k l, i} b_{k l, t} \\
+2 h_{t} \sigma_{1} \sum_{k} b^{k k} b_{k k, t}-4 h_{t} \sum_{k}\left(b^{k k}\right)^{2} b_{k k, t}-2 h_{t}^{2} \sigma_{1}^{2}+2 \sum_{k, l} b^{k k} b^{l l} b_{k l, t}^{2} \\
+\left[(n-1)\left(1+h_{t}^{2}\right)+2 h_{t}^{2}\right] \sum_{i}\left(b^{i i}\right)^{2}+2 \sigma_{1} \sum_{i} h_{t i}^{2} b^{i i} \\
+(n-3) \sum_{i}\left(h_{t i} b^{i i}\right)^{2} .
\end{array}
$$


By substituting (3-9) and (3-16) in (3-4), we obtain

$$
\begin{aligned}
L(\varphi)= & \sum_{i, j, k, l}\left[\left(1+h_{t}^{2}\right) \delta_{i j}+h_{t i} h_{t j}\right] b^{i i} b^{j j} b^{k k} b^{l l} b_{k l, i} b_{k l, j}-2 \sum_{i, k, l} h_{t i} b^{i i} b^{k k} b^{l l} b_{k l, i} b_{k l, t} \\
& +\sum_{k, l} b^{k k} b^{l l} b_{k l, t}^{2}+2 h_{t} \sigma_{1} \sum_{k} b^{k k} b_{k k, t}-4 h_{t} \sum_{k}\left(b^{k k}\right)^{2} b_{k k, t} \\
& +\left(2+4 \rho^{\prime} h_{t}^{2}\right) \sigma_{1} \sum_{i} h_{t i}^{2} b^{i i}+\left[(n-1)\left(1+h_{t}^{2}\right)+2 h_{t}^{2}-2 \rho^{\prime} h_{t}^{2}\left(1+h_{t}^{2}\right)\right] \sum_{i}\left(b^{i i}\right)^{2} \\
& +\left[4 \rho^{\prime} h_{t}^{2}\left(1+h_{t}^{2}\right)+\left(2 \rho^{\prime}+4 \rho^{\prime \prime} h_{t}^{2}\right)\left(1+h_{t}^{2}\right)^{2}-2 h_{t}^{2}\right] \sigma_{1}^{2} \\
& +\left[\left(2 \rho^{\prime}+4 \rho^{\prime \prime} h_{t}^{2}\right)\left(1+h_{t}^{2}\right)-2 \rho^{\prime} h_{t}^{2}+(n-3)\right] \sum_{i}\left(h_{t i} b^{i i}\right)^{2} .
\end{aligned}
$$

Step 2: calculation of $L\left(e^{\beta \varphi}\right)$ and estimation of the third-order derivatives involving $b_{k k, t}$. Notice that

$$
\begin{array}{r}
L\left(e^{\beta \varphi}\right)=\beta e^{\beta \varphi}\left\{L(\varphi)+\beta \varphi_{t}^{2}\right\}+\beta^{2} e^{\beta \varphi} \sum_{i, j, p, q}\left[\left(1+h_{t}^{2}\right) \delta_{p q}+h_{t p} h_{t q}\right] b^{i p} b^{j q} \frac{\partial \varphi}{\partial \theta_{i}} \frac{\partial \varphi}{\partial \theta_{j}} \\
-2 \beta^{2} e^{\beta \varphi} \sum_{i, j} h_{t j} b^{i j} \frac{\partial \varphi}{\partial \theta_{i}} \frac{\partial \varphi}{\partial t} .
\end{array}
$$

To reach (3-1), we only need to prove that, for some constant $\beta<0$,

$$
L(\varphi)+\beta \varphi_{t}^{2} \geq 0 \bmod \nabla_{\theta} \varphi .
$$

We now compute $\beta \varphi_{t}^{2}$.

By (3-3), we have

(3-18) $\varphi_{t}^{2}=4\left(\rho^{\prime}\right)^{2} h_{t}^{2} h_{t t}^{2}+4 \rho^{\prime} h_{t} h_{t t} \sum_{k} b^{k k} b_{k k, t}+\left(\sum_{k} b^{k k} b_{k k, t}\right)^{2}$

$$
\begin{aligned}
& =4\left(\rho^{\prime}\right)^{2} h_{t}^{2}\left(1+h_{t}^{2}\right)^{2} \sigma_{1}^{2}+8\left(\rho^{\prime}\right)^{2} h_{t}^{2}\left(1+h_{t}^{2}\right) \sigma_{1} \sum_{i} h_{t i}^{2} b^{i i} \\
& +4\left(\rho^{\prime}\right)^{2} h_{t}^{2}\left(\sum_{i} h_{t i}^{2} b^{i i}\right)^{2}+4 \rho^{\prime} h_{t}\left(1+h_{t}^{2}\right) \sigma_{1} \sum_{k} b^{k k} b_{k k, t} \\
& +4 \rho^{\prime} h_{t}\left(\sum_{i} h_{t i}^{2} b^{i i}\right)\left(\sum_{k} b^{k k} b_{k k, t}\right)+\left(\sum_{k} b^{k k} b_{k k, t}\right)^{2} .
\end{aligned}
$$

Joining (3-17) with (3-18), we regroup the terms in $L(\varphi)+\beta \varphi_{t}^{2}$ as follows:

$$
L(\varphi)+\beta \varphi_{t}^{2}=P_{1}+P_{2}+P_{3},
$$


where

$$
\begin{aligned}
& P_{1}= \sum_{k \neq l}\left(\sum_{i, j} h_{t i} h_{t j} b^{i i} b^{j j} b^{k k} b^{l l} b_{k l, i} b_{k l, j}-2 \sum_{i} h_{t i} b^{i i} b^{k k} b^{l l} b_{k l, i} b_{k l, t}\right. \\
&\left.+b^{k k} b^{l l} b_{k l, t}^{2}\right), \\
& P_{2}=\sum_{k}\left(b^{k k} b_{k k, t}\right)^{2}+\beta\left(\sum_{k} b^{k k} b_{k k, t}\right)^{2} \\
&+2 \sum_{k}\left[\left[1+2 \beta \rho^{\prime}\left(1+h_{t}^{2}\right)\right] h_{t} \sigma_{1}+2 \beta \rho^{\prime} h_{t}\left(\sum_{i} h_{t i}^{2} b^{i i}\right)\right. \\
&\left.\quad-\sum_{i} h_{t i} b^{i i} b^{k k} b_{k k, i}-2 h_{t} b^{k k}\right] \cdot\left(b^{k k} b_{k k, t}\right), \\
& P_{3}=\left(1+h_{t}^{2}\right) \sum_{i, k, l}\left(b^{i i}\right)^{2} b^{k k} b^{l l} b_{k l, i}^{2}+\sum_{i, j, k} h_{t i} h_{t j} b^{i i} b^{j j} b^{k k} b_{k k, i} b^{k k} b_{k k, j} \\
&+\left[2+4 \rho^{\prime} h_{t}^{2}+8 \beta\left(\rho^{\prime}\right)^{2} h_{t}^{2}\left(1+h_{t}^{2}\right)\right] \sigma_{1} \sum_{i} h_{t i}^{2} b^{i i} \\
&+\left[(n-1)\left(1+h_{t}^{2}\right)+2 h_{t}^{2}-2 \rho^{\prime} h_{t}^{2}\left(1+h_{t}^{2}\right)\right] \sum_{i}\left(b^{i i}\right)^{2} \\
&+\left[4 \rho^{\prime} h_{t}^{2}\left(1+h_{t}^{2}\right)+\left(2 \rho^{\prime}+4 \rho^{\prime \prime} h_{t}^{2}\right)\left(1+h_{t}^{2}\right)^{2}-2 h_{t}^{2}+4 \beta\left(\rho^{\prime}\right)^{2} h_{t}^{2}\left(1+h_{t}^{2}\right)^{2}\right] \sigma_{1}^{2} \\
&+\left[\left(2 \rho^{\prime}+4 \rho^{\prime \prime} h_{t}^{2}\right)\left(1+h_{t}^{2}\right)-2 \rho^{\prime} h_{t}^{2}+(n-3)\right] \sum_{i}\left(h_{t i} b^{i i}\right)^{2} \\
&+ 4 \beta\left(\rho^{\prime}\right)^{2} h_{t}^{2}\left(\sum_{i} h_{t i}^{2} b^{i i}\right)^{2} .
\end{aligned}
$$

In the rest of this step, we will deal with the term $P_{2}$. Let $X_{k}=b^{k k} b_{k k, t}(k=$ $1,2, \ldots, n-1)$. Then $P_{2}$ can be rewritten as

where

$$
P_{2}\left(X_{1}, X_{2}, \ldots, X_{n-1}\right)=\sum_{k} X_{k}^{2}+\beta\left(\sum_{k} X_{k}\right)^{2}+2 \sum_{k} c_{k} X_{k},
$$

$c_{k}=\left[1+2 \beta \rho^{\prime}\left(1+h_{t}^{2}\right)\right] h_{t} \sigma_{1}+2 \beta \rho^{\prime} h_{t}\left(\sum_{i} h_{t i}^{2} b^{i i}\right)-\sum_{i} h_{t i} b^{i i} b^{k k} b_{k k, i}-2 h_{t} b^{k k}$.

Denote by $\mathscr{P}_{2}$ the matrix

$$
\left(\begin{array}{cccc}
1+\beta & \beta & \cdots & \beta \\
\beta & 1+\beta & \cdots & \beta \\
\vdots & \vdots & \ddots & \cdots \\
\beta & \beta & \cdots & 1+\beta
\end{array}\right)
$$


In a word, we want to bound $P_{2}\left(X_{1}, X_{2}, \ldots, X_{n-1}\right)$ from below. Thus the nonnegativity of $\mathscr{P}_{2}$ is necessary, and this requires

$$
\beta \geq-\frac{1}{n-1} .
$$

For convenience, Let us choose the degenerate case, that is, $\beta=-1 /(n-1)$. By setting $\tau=(1,1, \ldots, 1)$, the null eigenvector of the matrix $\mathscr{P}_{2}$, we then have, by $(3-2)$,



which suggests that the simplest selection should be $\rho(t)=((n-3) / 2) \log (1+t)$.

From now on, let us fix $\rho(t)=((n-3) / 2) \log (1+t)$ and $\beta=-1 /(n-1)$. But, for simplicity, we do not always substitute for the values of $\rho$ and $\beta$.

By straightforward computation and $(\star)$, we have

$$
\sum_{k}\left(X_{k}+\beta \sum_{i} X_{i}+c_{k}\right)^{2}=P_{2}\left(X_{1}, X_{2}, \ldots, X_{n-1}\right)+\sum_{k} c_{k}^{2}+P_{2}\left(\nabla_{\theta} \varphi\right),
$$

where

$$
P_{2}\left(\nabla_{\theta} \varphi\right)=2 \beta\left(\sum_{i} X_{i}\right) \sum_{k} c_{k}=2 \beta\left(\sum_{j} X_{j}\right) \sum_{i} h_{t i} b^{i i} \frac{\partial \varphi}{\partial \theta_{i}} .
$$

Putting $\rho$ and $\beta$ into some terms in $c_{k}$, we derive that

$$
c_{k}=\frac{2}{n-1} h_{t} \sigma_{1}-\frac{2}{n-1} \rho^{\prime} h_{t}\left(\sum_{i} h_{t i}^{2} b^{i i}\right)-\sum_{i} h_{t i} b^{i i} b^{k k} b_{k k, i}-2 h_{t} b^{k k} .
$$

Therefore, together with (3-2), we get

$$
\begin{aligned}
& P_{2}\left(X_{1}, X_{2}, \ldots, X_{n-1}\right) \\
& \geq-\sum_{k} c_{k}^{2}-P_{2}\left(\nabla_{\theta} \varphi\right) \\
& =-\sum_{i, j, k} h_{t i} h_{t j} b^{i i} b^{j j} b^{k k} b_{k k, i} b^{k k} b_{k k, j}-4 h_{t} \sum_{i, k} h_{t i} b^{i i}\left(b^{k k}\right)^{2} b_{k k, i} \\
& -4 h_{t}^{2} \sum_{k}\left(b^{k k}\right)^{2}+\frac{4}{n-1} h_{t}^{2} \sigma_{1}^{2}-\frac{8}{n-1} \rho^{\prime} h_{t}^{2} \sigma_{1} \sum_{i} h_{t i}^{2} b^{i i} \\
& +\frac{4}{n-1} h_{t}^{2}\left(\rho^{\prime}\right)^{2}\left(\sum_{i} h_{t i}^{2} b^{i i}\right)^{2}+\widetilde{P}_{2}\left(\nabla_{\theta} \varphi\right),
\end{aligned}
$$

where

$$
\widetilde{P}_{2}\left(\nabla_{\theta} \varphi\right)=-P_{2}\left(\nabla_{\theta} \varphi\right)-\frac{4}{n-1} h_{t}\left[\sigma_{1}-\rho^{\prime} \sum_{j} h_{t j}^{2} b^{j j}\right] \sum_{i} h_{t i} b^{i i} \frac{\partial \varphi}{\partial \theta_{i}} .
$$


Observing that $P_{1} \geq 0$,

$$
\begin{aligned}
& L(\varphi)+\beta \varphi_{t}^{2} \\
& \begin{array}{c}
\geq\left(1+h_{t}^{2}\right) \sum_{i, k, l}\left(b^{i i}\right)^{2} b^{k k} b^{l l} b_{k l, i}^{2}-4 h_{t} \sum_{i, k} h_{t i} b^{i i}\left(b^{k k}\right)^{2} b_{k k, i} \\
+\left[2+4 \rho^{\prime} h_{t}^{2}+8 \beta\left(\rho^{\prime}\right)^{2} h_{t}^{2}\left(1+h_{t}^{2}\right)-\frac{8}{n-1} \rho^{\prime} h_{t}^{2}\right] \sigma_{1} \sum_{i} h_{t i}^{2} b^{i i} \\
+\left[(n-1)\left(1+h_{t}^{2}\right)-2 h_{t}^{2}-2 \rho^{\prime} h_{t}^{2}\left(1+h_{t}^{2}\right)\right] \sum_{i}\left(b^{i i}\right)^{2} \\
+\left[4 \rho^{\prime} h_{t}^{2}\left(1+h_{t}^{2}\right)+\left[\left(2 \rho^{\prime}+4 \rho^{\prime \prime} h_{t}^{2}\right)+4 \beta\left(\rho^{\prime}\right)^{2} h_{t}^{2}\right]\left(1+h_{t}^{2}\right)^{2}-\frac{2 n-6}{n-1} h_{t}^{2}\right] \sigma_{1}^{2} \\
+\left[\left(2 \rho^{\prime}+4 \rho^{\prime \prime} h_{t}^{2}\right)\left(1+h_{t}^{2}\right)-2 \rho^{\prime} h_{t}^{2}+(n-3)\right] \sum_{i}\left(h_{t i} b^{i i}\right)^{2}+\widetilde{P}_{2}\left(\nabla_{\theta} \varphi\right) .
\end{array}
\end{aligned}
$$

In the next step we will concentrate on the following two terms:

$$
R=\left(1+h_{t}^{2}\right) \sum_{i, k, l}\left(b^{i i}\right)^{2} b^{k k} b^{l l} b_{k l, i}^{2}-4 h_{t} \sum_{i, k} h_{t i} b^{i i}\left(b^{k k}\right)^{2} b_{k k, i} .
$$

Step 3: conclusion of the proof of (3-1). Recalling our first-order condition (3-2), we have

$$
b^{11} b_{11, j}=\frac{\partial \varphi}{\partial \theta_{j}}-\sum_{k \geq 2} b^{k k} b_{k k, j}-2 \rho^{\prime} h_{t} h_{t j} \quad \text { for } j=1,2, \ldots, n-1 .
$$

For the term $R$, we have

$$
\begin{gathered}
R=\left(1+h_{t}^{2}\right)\left[\sum_{i} \sum_{k \neq l}\left(b^{i i}\right)^{2} b^{k k} b^{l l} b_{k l, i}^{2}+\sum_{i, k}\left(b^{i i}\right)^{2}\left(b^{k k} b_{k k, i}\right)^{2}\right] \\
-4 \sum_{i, k} h_{t} h_{t i} b^{i i}\left(b^{k k}\right)^{2} b_{k k, i} \\
=\left(1+h_{t}^{2}\right)\left[2 \sum_{k \geq 2}\left(b^{11}\right)^{2} b^{k k} b^{11} b_{k 1,1}^{2}+2 \sum_{i, k \geq 2}\left(b^{i i}\right)^{2} b^{k k} b^{11} b_{k 1, i}^{2}\right. \\
+\sum_{i} \sum_{\substack{k, l \geq 2 \\
k \neq l}}\left(b^{i i}\right)^{2} b^{k k} b^{l l} b_{k l, i}^{2}+\sum_{i}\left(b^{i i}\right)^{2}\left(b^{11} b_{11, i}\right)^{2} \\
\left.+\sum_{i} \sum_{k \geq 2}\left(b^{i i}\right)^{2}\left(b^{k k} b_{k k, i}\right)^{2}\right] \\
-4 \sum_{i} h_{t} h_{t i} b^{i i}\left(b^{11}\right)^{2} b_{11, i}-4 \sum_{i} \sum_{k \geq 2} h_{t} h_{t i} b^{i i}\left(b^{k k}\right)^{2} b_{k k, i} \\
=R_{1}+R_{2}+R_{3}, \quad
\end{gathered}
$$


where

$$
\begin{aligned}
R_{1}= & \left(1+h_{t}^{2}\right)\left[2 \sum_{k \geq 2}\left(b^{11}\right)^{2} b^{k k} b^{11} b_{k 1,1}^{2}+\sum_{i}\left(b^{i i}\right)^{2}\left(b^{11} b_{11, i}\right)^{2}\right] \\
& -4 \sum_{i} h_{t} h_{t i} b^{i i}\left(b^{11}\right)^{2} b_{11, i}, \\
R_{2}= & 2 \sum_{i, k \geq 2}\left(1+h_{t}^{2}\right)\left(b^{i i}\right)^{2} b^{k k} b^{11} b_{k 1, i}^{2}+\sum_{i} \sum_{\substack{k, l \geq 2 \\
k \neq l}}\left(1+h_{t}^{2}\right)\left(b^{i i}\right)^{2} b^{k k} b^{l l} b_{k l, i}^{2}, \\
R_{3}= & \sum_{i} \sum_{k \geq 2}\left(1+h_{t}^{2}\right)\left(b^{i i}\right)^{2}\left(b^{k k} b_{k k, i}\right)^{2}-4 \sum_{i} \sum_{k \geq 2} h_{t} h_{t i} b^{i i}\left(b^{k k}\right)^{2} b_{k k, i} .
\end{aligned}
$$

By (3-20), one has

$$
\begin{aligned}
R_{1}=\left(1+h_{t}^{2}\right)\left[2 b^{11} \sum_{i, k, l \geq 2} b^{i i} b^{k k} b^{l l} b_{k k, i} b_{l l, i}+8 \rho^{\prime} h_{t} b^{11} \sum_{i, k \geq 2} h_{t i} b^{i i} b^{k k} b_{k k, i}\right. \\
+8\left(\rho^{\prime}\right)^{2} h_{t}^{2} b^{11} \sum_{i \geq 2} h_{t i}^{2} b^{i i}+\sum_{i} \sum_{k, l \geq 2}\left(b^{i i}\right)^{2} b^{k k} b^{l l} b_{k k, i} b_{l l, i} \\
\left.\quad+4 \rho^{\prime} h_{t} \sum_{i} \sum_{k \geq 2} h_{t i}\left(b^{i i}\right)^{2} b^{k k} b_{k k, i}+4\left(\rho^{\prime}\right)^{2} h_{t}^{2} \sum_{i}\left(h_{t i} b^{i i}\right)^{2}\right] \\
+4 h_{t} \sum_{i} \sum_{k \geq 2} h_{t i} b^{i i} b^{11} b^{k k} b_{k k, i}+8 \rho^{\prime} h_{t}^{2} b^{11} \sum_{i} h_{t i}^{2} b^{i i}+R\left(\nabla_{\theta} \varphi\right),
\end{aligned}
$$

where

$$
\begin{array}{r}
R\left(\nabla_{\theta} \varphi\right)=\left(1+h_{t}^{2}\right)\left[2 b^{11} \sum_{k \geq 2} b^{k k}\left(\frac{\partial \varphi}{\partial \theta_{k}}\right)^{2}-4 b^{11} \sum_{k, l \geq 2} b^{k k} b^{l l} b_{l l, k} \frac{\partial \varphi}{\partial \theta_{k}}\right. \\
-8 \rho^{\prime} h_{t} b^{11} \sum_{k \geq 2} b^{k k} h_{t k} \frac{\partial \varphi}{\partial \theta_{k}}+\sum_{i}\left(b^{i i}\right)^{2}\left(\frac{\partial \varphi}{\partial \theta_{i}}\right)^{2} \\
\left.-2 \sum_{i} \sum_{k \geq 2}\left(b^{i i}\right)^{2} b^{k k} b_{k k, i} \frac{\partial \varphi}{\partial \theta_{i}}-4 \rho^{\prime} h_{t} \sum_{i}\left(b^{i i}\right)^{2} h_{t i} \frac{\partial \varphi}{\partial \theta_{i}}\right] \\
-4 h_{t} b^{11} \sum_{i} b^{i i} h_{t i} \frac{\partial \varphi}{\partial \theta_{i}} .
\end{array}
$$

On the other hand,

$$
\begin{gathered}
R_{2}=\left(1+h_{t}^{2}\right)\left[2 b^{11} \sum_{k \geq 2}\left(b^{k k}\right)^{3} b_{k k, 1}^{2}+2 \sum_{\substack{i, k \geq 2 \\
i \neq k}}\left(b^{i i}\right)^{2} b^{k k} b^{11} b_{k 1, i}^{2}\right. \\
\left.\quad+2 \sum_{\substack{i, k \geq 2 \\
i \neq k}} b^{i i}\left(b^{k k}\right)^{3} b_{k k, i}^{2}+\sum_{i} \sum_{\substack{k, l \geq 2 \\
k \neq l, k \neq i, l \neq i}}\left(b^{i i}\right)^{2} b^{k k} b^{l l} b_{k l, i}^{2}\right] .
\end{gathered}
$$


Recall that $2 \rho^{\prime}\left(1+h_{t}^{2}\right)=n-3$, which will be denoted by $\alpha$ for simplicity in the following calculations. Now we are at a stage where we can rewrite the terms in $R$ in a natural way: we denote by $T_{1}$ the terms involving $b_{k k, 1}(k \geq 2)$, by $T_{2}$ the terms involving $b_{k k, i}(k, i \geq 2)$, and by $T_{3}$ all of the rest of the terms. More precisely,

$$
\begin{array}{r}
T_{1}=\sum_{k \geq 2}\left(1+2 b_{11} b^{k k}\right) \cdot\left(\left(1+h_{t}^{2}\right)^{1 / 2} b^{11} b^{k k} b_{k k, 1}\right)^{2}+\left(\sum_{k \geq 2}\left(1+h_{t}^{2}\right)^{1 / 2} b^{11} b^{k k} b_{k k, 1}\right)^{2} \\
\quad+4 h_{t} h_{t 1} b^{11}\left(1+h_{t}^{2}\right)^{-1 / 2} \sum_{k \geq 2}\left(1+\frac{\alpha}{2}-b_{11} b^{k k}\right) \cdot\left(\left(1+h_{t}^{2}\right)^{1 / 2} b^{11} b^{k k} b_{k k, 1}\right)
\end{array}
$$

and

$$
\begin{aligned}
& T_{2}=\left(1+h_{t}^{2}\right) \sum_{i \geq 2}\{(1+\left.2 b_{i i} b^{11}\right) \cdot\left(\sum_{k \geq 2} b^{i i} b^{k k} b_{k k, i}\right)^{2}+\sum_{\substack{k \geq 2 \\
k \neq i}} 2 b_{i i} b^{k k} \cdot\left(b^{i i} b^{k k} b_{k k, i}\right)^{2} \\
&+\sum_{k \geq 2}\left(b^{i i} b^{k k} b_{k k, i}\right)^{2}+4 h_{t} h_{t i} b^{i i}\left(1+h_{t}^{2}\right)^{-1} \\
&\left.\times \sum_{k \geq 2}\left[-b_{i i} b^{k k}+\frac{\alpha}{2}+(1+\alpha) b_{i i} b^{11}\right] \cdot\left(b^{i i} b^{k k} b_{k k, i}\right)\right\} ;
\end{aligned}
$$

the rest of the terms are

$$
\begin{array}{r}
T_{3}=h_{t}^{2}\left(1+h_{t}^{2}\right)^{-1}\left[2 \alpha^{2} b^{11} \sum_{i \geq 2} h_{t i}^{2} b^{i i}+\alpha^{2} \sum_{i}\left(h_{t i} b^{i i}\right)^{2}+4 \alpha b^{11} \sum_{i} h_{t i}^{2} b^{i i}\right] \\
+\left(1+h_{t}^{2}\right)\left[2 \sum_{\substack{i, k \geq 2 \\
i \neq k}}\left(b^{i i}\right)^{2} b^{k k} b^{11} b_{k 1, i}^{2}+\sum_{i} \sum_{\substack{k, l \geq 2 \\
k \neq l, k \neq i, l \neq i}}\left(b^{i i}\right)^{2} b^{k k} b^{l l} b_{k l, i}^{2}\right] \\
+R\left(\nabla_{\theta} \varphi\right) .
\end{array}
$$

We shall minimize the terms $T_{1}$ and $T_{2}$ via Lemma 3.1 for different choices of parameters.

At first, let us examine the term $T_{1}$. set $X_{k}=\left(1+h_{t}^{2}\right)^{1 / 2} b^{11} b^{k k} b_{k k, 1}, \lambda=1$, $\mu=h_{t 1} b^{11} h_{t}\left(1+h_{t}^{2}\right)^{-1 / 2}, b_{k}=1+2 b_{11} b^{k k}$, and $c_{k}=b_{11} b^{k k}-(1+\alpha / 2)$, where $k \geq 2$. By Lemma 3.1, we have

$$
T_{1} \geq-4 h_{t}^{2}\left(1+h_{t}^{2}\right)^{-1}\left(h_{t 1} b^{11}\right)^{2} \Gamma_{1},
$$

where

$$
\Gamma_{1}=\sum_{k \geq 2} \frac{c_{k}^{2}}{b_{k}}-\left(1+\sum_{k \geq 2} \frac{1}{b_{k}}\right)^{-1}\left(\sum_{k \geq 2} \frac{c_{k}}{b_{k}}\right)^{2} .
$$

Next we shall simplify $\Gamma_{1}$. By denoting

$$
\beta_{k}=\frac{1}{b_{k}}
$$


we have

$$
b_{11} b^{k k}=\frac{1}{2 \beta_{k}}-\frac{1}{2}, \quad c_{k}=\frac{1}{2 \beta_{k}}-\frac{3+\alpha}{2} .
$$

Hence

$$
\begin{aligned}
\Gamma_{1} & =\sum_{k \geq 2} \beta_{k}\left(\frac{1}{2 \beta_{k}}-\frac{3+\alpha}{2}\right)^{2}-\left(1+\sum_{k \geq 2} \beta_{k}\right)^{-1}\left[\sum_{k \geq 2} \beta_{k}\left(\frac{1}{2 \beta_{k}}-\frac{3+\alpha}{2}\right)\right]^{2} \\
& =\frac{1}{4} \sum_{k \geq 2} \frac{1}{\beta_{k}}-\left(1+\sum_{k \geq 2} \beta_{k}\right)^{-1} \frac{(n+1+\alpha)^{2}}{4}+\frac{(3+\alpha)^{2}}{4} .
\end{aligned}
$$

Since

it follows that

$$
1 \leq 1+\sum_{k \geq 2} \beta_{k} \leq n-1
$$

$$
\begin{aligned}
\Gamma_{1} & \leq \frac{1}{4} \sum_{k \geq 2} \frac{1}{\beta_{k}}-\frac{(n+1+\alpha)^{2}}{4(n-1)}+\frac{(3+\alpha)^{2}}{4} \\
& =\frac{n-2}{4(n-1)}(2+\alpha)^{2}+\frac{1}{4}\left(2 \sigma_{1} b_{11}-2\right) .
\end{aligned}
$$

Therefore,

$$
T_{1} \geq-\left[\frac{(n-2)}{n-1}(2+\alpha)^{2}+2 \sigma_{1} b_{11}-2\right] h_{t}^{2}\left(1+h_{t}^{2}\right)^{-1}\left(h_{t 1} b^{11}\right)^{2} .
$$

Now we will deal with $T_{2}$. For every $i \geq 2$ fixed, set $X_{k}=b^{i i} b^{k k} b_{k k, i}, \lambda=$ $1+2 b_{i i} b^{11}, \mu=-h_{t i} b^{i i} h_{t}\left(1+h_{t}^{2}\right)^{-1}, b_{k}=1+2 b_{i i} b^{k k}(k \neq i), b_{i}=1$, and $c_{k}=$ $b_{i i} b^{k k}-\frac{1}{2} \alpha-(1+\alpha) b_{i i} b^{11}$. By Lemma 3.1, we have

$$
T_{2} \geq-4\left(1+h_{t}^{2}\right) \sum_{i \geq 2}\left(h_{t i} b^{i i}\right)^{2} \Gamma_{i}
$$

where

$$
\Gamma_{i}=c_{i}^{2}+\sum_{\substack{k \geq 2 \\ k \neq i}} \frac{c_{k}^{2}}{b_{k}}-\left(\frac{1}{\lambda}+1+\sum_{\substack{k \geq 2 \\ k \neq i}} \frac{1}{b_{k}}\right)^{-1}\left(c_{i}+\sum_{\substack{k \geq 2 \\ k \neq i}} \frac{c_{k}}{b_{k}}\right)^{2} .
$$

For $k \neq i$, denoting

$$
\beta_{k}=\frac{1}{b_{k}}
$$

we have

$$
b_{i i} b^{k k}=\frac{1}{2 \beta_{k}}-\frac{1}{2}, \quad c_{k}=\frac{1}{2 \beta_{k}}-\delta,
$$

where

$$
\delta=\frac{1+\alpha}{2}+(1+\alpha) b_{i i} b^{11} .
$$


Noticing that

$$
c_{i}=\frac{3}{2}-\delta, \quad \frac{\delta}{\lambda}=\frac{1+\alpha}{2},
$$

we obtain

$$
\begin{aligned}
\Gamma_{i} & =c_{i}^{2}+\sum_{\substack{k \geq 2 \\
k \neq i}} \beta_{k}\left(\frac{1}{2 \beta_{k}}-\delta\right)^{2}-\left(\frac{1}{\lambda}+1+\sum_{\substack{k \geq 2 \\
k \neq i}} \beta_{k}\right)^{-1}\left[c_{i}+\sum_{\substack{k \geq 2 \\
k \neq i}} \beta_{k}\left(\frac{1}{2 \beta_{k}}-\delta\right)\right]^{2} \\
& =\frac{1}{4} \sum_{\substack{k \geq 2 \\
k \neq i}} \frac{1}{\beta_{k}}-\left(\frac{1}{\lambda}+1+\sum_{\substack{k \geq 2 \\
k \neq i}} \beta_{k}\right)^{-1}\left(\frac{n}{2}+\frac{\delta}{\lambda}\right)^{2}+\frac{9}{4}+\frac{\delta^{2}}{\lambda} \\
& =\frac{1}{4} \sum_{\substack{k \geq 2 \\
k \neq i}} \frac{1}{\beta_{k}}-\left(\frac{1}{\lambda}+1+\sum_{\substack{k \geq 2 \\
k \neq i}} \beta_{k}\right)^{-1} \frac{(n+1+\alpha)^{2}}{4}+\frac{9}{4}+\frac{1+\alpha}{2} \delta .
\end{aligned}
$$

Obviously,

hence

$$
1 \leq \frac{1}{\lambda}+1+\sum_{\substack{k \geq 2 \\ k \neq i}} \beta_{k} \leq n-1,
$$

$$
\begin{aligned}
\Gamma_{i} & \leq \frac{1}{4} \sum_{\substack{k \geq 2 \\
k \neq i}} \frac{1}{\beta_{k}}-\frac{(n+1+\alpha)^{2}}{4(n-1)}+\frac{9}{4}+\frac{1+\alpha}{2} \delta \\
& =\frac{n-2}{4(n-1)} \alpha^{2}-\frac{1}{n-1} \alpha+\frac{n-3}{2(n-1)}+\frac{1}{2} \sigma_{1} b_{i i}+\frac{1}{2} \alpha^{2} b_{i i} b^{11}+\alpha b_{i i} b^{11} .
\end{aligned}
$$

Therefore, we have

$$
\begin{aligned}
T_{2} \geq-\frac{h_{t}^{2}}{1+h_{t}^{2}} \sum_{i \geq 2}\left(\frac{n-2}{n-1} \alpha^{2}-\right. & \frac{4}{n-1} \alpha+\frac{2 n-6}{n-1} \\
& \left.+2 \sigma_{1} b_{i i}+2 \alpha^{2} b_{i i} b^{11}+4 \alpha b_{i i} b^{11}\right)\left(h_{t i} b^{i i}\right)^{2} .
\end{aligned}
$$

Now, combining (3-21), (3-22), and (3-23), we obtain

$$
R \geq \frac{h_{t}^{2}}{1+h_{t}^{2}} \sum_{i}\left(\frac{1}{n-1} \alpha^{2}+\frac{4}{n-1} \alpha-\frac{2 n-6}{n-1}-2 \sigma_{1} b_{i i}\right)\left(h_{t i} b^{i i}\right)^{2}+R\left(\nabla_{\theta} \varphi\right)
$$

For choices of $\rho$ and $\beta$, by (3-19) and (3-24), we have, for $n \geq 2$,

$$
\begin{aligned}
L(\varphi)-\frac{1}{n-1} \varphi_{t}^{2} & \geq \frac{2 \sigma_{1}}{1+h_{t}^{2}} \sum_{i} h_{t i}^{2} b^{i i}+(n-1) \sum_{i}\left(b^{i i}\right)^{2}+(n-3) \sigma_{1}^{2} \\
& +\frac{2(n-3)}{1+h_{t}^{2}} \sum_{i}\left(h_{t i} b^{i i}\right)^{2}+\widetilde{P}_{2}\left(\nabla_{\theta} \varphi\right)+R\left(\nabla_{\theta} \varphi\right) \\
\geq & 0 \bmod \nabla_{\theta} \varphi .
\end{aligned}
$$


The proof of (3-1) is completed.

Now we give a remark on Theorem 1.1.

Remark 3.3. In the proof of Theorem 1.1, if we restrict to the case $n=2$ and just set $\rho=0$, then (3-2) shows that

$$
b_{11,1}=0 \quad \bmod \nabla_{\theta} \varphi .
$$

Applying this to the expression of $L(\varphi)$ in (3-17) will give

$$
\begin{aligned}
L(\varphi) & =\left(b^{11} b_{11, t}\right)^{2}-2 h_{t}\left(b^{11}\right)^{2} b_{11, t}+\left(b^{11}\right)^{2} h_{t 1}^{2}+\left(1+h_{t}^{2}\right)\left(b^{11}\right)^{2} \\
& =\left[b^{11} b_{11, t}-h_{t} b^{11}\right]^{2}+\left(b^{11}\right)^{2} h_{t 1}^{2}+\left(b^{11}\right)^{2} \geq 0 \bmod \nabla_{\theta} \varphi,
\end{aligned}
$$

and this means that, for any point $x \in \Gamma_{t}, 0<t<1$,

$$
\log K(x) \geq(1-t) \min _{\partial \Omega_{0}} \log K+t \min _{\partial \Omega_{1}} \log K,
$$

which has already been proved by Longinetti [1987]. Also, by Remark 1.3 we know that this estimate is not sharp in the two-dimensional case.

\section{Acknowledgments}

The author thanks Professor X. Ma for many useful discussions on this subject, and the School of Mathematical Sciences of University of Sciences and Technology of China for hospitality.

The author also thanks the referees for their careful efforts to make the paper clearer.

Part of this work was done while the first author was staying at his postdoctoral mobile research station in QFNU.

\section{References}

[Caffarelli and Spruck 1982] L. A. Caffarelli and J. Spruck, "Convexity properties of solutions to some classical variational problems", Comm. Partial Differential Equations 7:11 (1982), 1337-1379. MR 85f:49062 Zbl 0508.49013

[Chen and Shi 2011] C. Chen and S. Shi, "Curvature estimates for the level sets of spatial quasiconcave solutions to a class of parabolic equations", Sci. China Math. 54:10 (2011), 2063-2080. MR 2838121

[Chiti and Longinetti 1992] G. Chiti and M. Longinetti, "Differential inequalities for Minkowski functionals of level sets", pp. 109-127 in General inequalities, 6 (Oberwolfach, 1990), edited by W. Walter, Internat. Ser. Numer. Math. 103, Birkhäuser, Basel, 1992. MR 94b:49073 Zbl 0764.53005

[Colesanti and Salani 2003] A. Colesanti and P. Salani, "The Brunn-Minkowski inequality for $p$ capacity of convex bodies", Math. Ann. 327:3 (2003), 459-479. MR 2004j:31007 Zbl 1052.31005

[Jost et al. 2012] J. Jost, X.-N. Ma, and Q. Ou, "Curvature estimates in dimensions 2 and 3 for the level sets of $p$-harmonic functions in convex rings", Trans. Amer. Math. Soc. 364:9 (2012), 4605-4627. MR 2922603 Zbl 06191423 
[Longinetti 1987] M. Longinetti, "On minimal surfaces bounded by two convex curves in parallel planes”, J. Differential Equations 67:3 (1987), 344-358. MR 88m:58035 Zbl 0626.53002

[Longinetti and Salani 2007] M. Longinetti and P. Salani, "On the Hessian matrix and Minkowski addition of quasiconvex functions", J. Math. Pures Appl. (9) 88:3 (2007), 276-292. MR 2008k:35163 Zbl 1144.26017

[Ma et al. 2010] X.-N. Ma, Q. Ou, and W. Zhang, "Gaussian curvature estimates for the convex level sets of p-harmonic functions", Comm. Pure Appl. Math. 63:7 (2010), 935-971. MR 2011d:35168 Zbl 1193.35031

[Ma et al. 2011] X.-N. Ma, J. Ye, and Y.-H. Ye, "Principal curvature estimates for the level sets of harmonic functions and minimal graph in $\mathbb{R}^{3 ",}$ Comm. Pure Appl. Anal. 10:1 (2011), 225-243. MR 2012d:35134 Zbl 1235.35083

[Schneider 1993] R. Schneider, Convex bodies: the Brunn-Minkowski theory, Encyclopedia of Mathematics and its Applications 44, Cambridge University Press, 1993. MR 94d:52007 Zbl 0798.52001

[Wang and Zhang 2012] P. Wang and W. Zhang, "Gaussian curvature estimates for the convex level sets of solutions for some nonlinear elliptic partial differential equations", J. Partial Differ. Equ. 25:3 (2012), 239-275. MR 2987367 Zbl 06178153 arXiv 1003.2057

Received February 16, 2012. Revised June 10, 2013.

PEI-HE WANG

SCHOOL OF Mathematical SCIENCES

QUFU NORMAL UNIVERSITY

Qufu, 273165, SHANDONG PROVINCE

CHINA

peihewang@hotmail.com 


\title{
PACIFIC JOURNAL OF MATHEMATICS
}

\author{
msp.org/pjm
}

Founded in 1951 by E. F. Beckenbach (1906-1982) and F. Wolf (1904-1989)

\section{EDITORS}

V. S. Varadarajan (Managing Editor)

Department of Mathematics

University of California

Los Angeles, CA 90095-1555

pacific@math.ucla.edu

Paul Balmer

Department of Mathematics

University of California

Los Angeles, CA 90095-1555

balmer@math.ucla.edu

Daryl Cooper

Department of Mathematics

University of California

Santa Barbara, CA 93106-3080 cooper@math.ucsb.edu

Jiang-Hua $\mathrm{Lu}$

Department of Mathematics

Pokfulam Rd., Hong Kong jhlu@maths.hku.hk
The University of Hong Kong

Don Blasius

Department of Mathematics University of California

Los Angeles, CA 90095-1555

blasius@math.ucla.edu

Robert Finn

Department of Mathematics Stanford University

Stanford, CA 94305-2125

finn@math.stanford.edu

Sorin Popa

Department of Mathematics

University of California

Los Angeles, CA 90095-1555 popa@math.ucla.edu

Paul Yang

Department of Mathematics Princeton University

Princeton NJ 08544-1000

yang@math.princeton.edu

\section{PRODUCTION}

Silvio Levy, Scientific Editor, production@msp.org

\section{SUPPORTING INSTITUTIONS}

ACADEMIA SINICA, TAIPEI

CALIFORNIA INST. OF TECHNOLOGY

INST. DE MATEMÁTICA PURA E APLICADA

KEIO UNIVERSITY

MATH. SCIENCES RESEARCH INSTITUTE

NEW MEXICO STATE UNIV.

OREGON STATE UNIV.

\author{
STANFORD UNIVERSITY \\ UNIV. OF BRITISH COLUMBIA \\ UNIV. OF CALIFORNIA, BERKELEY \\ UNIV. OF CALIFORNIA, DAVIS \\ UNIV. OF CALIFORNIA, LOS ANGELES \\ UNIV. OF CALIFORNIA, RIVERSIDE \\ UNIV. OF CALIFORNIA, SAN DIEGO \\ UNIV. OF CALIF., SANTA BARBARA
}

\author{
Vyjayanthi Chari \\ Department of Mathematics \\ University of California \\ Riverside, CA 92521-0135 \\ chari@math.ucr.edu \\ Kefeng Liu \\ Department of Mathematics \\ University of California \\ Los Angeles, CA 90095-1555 \\ liu@math.ucla.edu \\ Jie Qing \\ Department of Mathematics \\ University of California \\ Santa Cruz, CA 95064 \\ qing@cats.ucsc.edu
}

These supporting institutions contribute to the cost of publication of this Journal, but they are not owners or publishers and have no responsibility for its contents or policies.

See inside back cover or msp.org/pjm for submission instructions.

The subscription price for 2014 is US \$410/year for the electronic version, and \$535/year for print and electronic.

Subscriptions, requests for back issues and changes of subscribers address should be sent to Pacific Journal of Mathematics, P.O. Box 4163, Berkeley, CA 94704-0163, U.S.A. The Pacific Journal of Mathematics is indexed by Mathematical Reviews, Zentralblatt MATH, PASCAL CNRS Index, Referativnyi Zhurnal, Current Mathematical Publications and Web of Knowledge (Science Citation Index).

The Pacific Journal of Mathematics (ISSN 0030-8730) at the University of California, c/o Department of Mathematics, 798 Evans Hall \#3840, Berkeley, CA 94720-3840, is published twelve times a year. Periodical rate postage paid at Berkeley, CA 94704, and additional mailing offices. POSTMASTER: send address changes to Pacific Journal of Mathematics, P.O. Box 4163, Berkeley, CA 94704-0163.

PJM peer review and production are managed by EditFLOW ${ }^{\circledR}$ from Mathematical Sciences Publishers.

\section{PUBLISHED BY}

mathematical sciences publishers

nonprofit scientific publishing

http://msp.org/

(C) 2014 Mathematical Sciences Publishers 


\section{PACIFIC JOURNAL OF MATHEMATICS}

Volume $267 \quad$ No. $2 \quad$ February 2014

Sums of squares in algebraic function fields over a complete discretely valued field

KARIM JOHANNES BECHER, DAVID GRIMM and JAN VAN GEEL

On the equivalence problem for toric contact structures on $\mathbf{S}^{\mathbf{3}}$-bundles over $S^{2}$

\section{Charles P. Boyer and Justin PATi}

An almost-Schur type lemma for symmetric $(2,0)$ tensors and applications

Xu CHENG

Algebraic invariants, mutation, and commensurability of link complements

ERIC CHESEBRO and JASON DEBLOIS

Taut foliations and the action of the fundamental group on leaf spaces and universal circles

YOSUKE KANO

A new monotone quantity along the inverse mean curvature flow in $\mathbb{R}^{n}$

KwoK-Kun Kwong and Pengzi Miao

Nonfibered L-space knots

TYE LIDMAN and LIAM WATSON

Families and Springer's correspondence

GEORGE LUSZTIG

Reflexive operator algebras on Banach spaces

Florence Merlevède, Costel Peligrad and Magda Peligrad Harer stability and orbifold cohomology

NiCOLA PAGANI

Spectra of product graphs and permanents of matrices over finite rings

LE ANH VINH

The concavity of the Gaussian curvature of the convex level sets of minimal surfaces with respect to the height

PEI-HE WANG 